\title{
Chinois en Polynésie française : Migration, métissage, diasporade Anne-Christine TRÉMON
}

Benoît Vulliet

\section{OpenEdition}

Journals

Édition électronique

URL : http://journals.openedition.org/jso/6448

DOI : $10.4000 /$ jso.6448

ISSN : $1760-7256$

Éditeur

Société des océanistes

Édition imprimée

Date de publication : 15 décembre 2011

Pagination : 431-434

ISBN : 978-2-85430-031-4

ISSN : 0300-953x

\section{Référence électronique}

Benoît Vulliet, "Chinois en Polynésie française : Migration, métissage, diasporade Anne-Christine tRémon », Journal de la Société des Océanistes [En ligne], 133| 2e semestre 2011, mis en ligne le 31 décembre 2011, consulté le 21 septembre 2020. URL : http://journals.openedition.org/jso/6448 ; DOI : https:// doi.org/10.4000/jso.6448

Ce document a été généré automatiquement le 21 septembre 2020.

(c) Tous droits réservés 


\title{
Chinois en Polynésie française : Migration, métissage, diasporade Anne-Christine TRÉMON
}

\author{
Benoît Vulliet
}

\section{RÉFÉRENCE}

TRÉMON Anne-Christine, 2010. Chinois en Polynésie française : Migration, métissage, diaspora, Nanterre, Société d'ethnologie, 425 pages, bibliogr., 15 cartes et schémas, 15 photos couleur et noir et blanc hors texte.

Depuis quelques années, la Chine fait beaucoup parler d'elle, et les ouvrages concernant la présence chinoise hors de ses frontières se sont multipliés, et bon nombre d'idées fausses ont été colportées, comme l'accaparement des terres ou la déstructuration des marchés locaux. Avec son ouvrage sur les Chinois de Polynésie française, AnneChristine Trémon nous fournit un témoignage exemplaire tant sur la forme que le fond. Cette anthropologie de la présence chinoise en Polynésie française propose une approche historique, tant génétique que chronologique, diachronique et synchronique : importance de l'histoire ancienne et son incidence sur la réalité actuelle, présentation des faits marquants au fil du temps, évolution de l'adaptation des individus et des groupes, compréhension des dimensions économiques, sociales, juridiques et politiques de la situation que vit aujourd'hui la Polynésie française.

2 Après une introduction assez longue, l'ouvrage, dense et complexe, se décompose en trois parties, chacune subdivisée en trois grands chapitres, eux-mêmes subdivisés en deux ou trois sous-chapitres, et se termine par une conclusion, assez brève, suivie d'une intéressante liste des personnes citées comprenant aussi un index des citations et les dates des entretiens, puis de cartes et enfin d'une importante bibliographie présentée selon les thématiques abordées. Quinze photographies complètent l'ouvrage en son milieu. Sinon terminologique, un index des auteurs cités aurait été utile. 
3 Cette recherche a été conduite lors de quatre séjours en Polynésie française (13 mois) au cours desquels l'auteur a examiné les journaux locaux et métropolitains, mené 108 entretiens, fait de l'observation participante aussi bien dans les familles que dans les magasins, manifestations culturelles ou tout autre réunion (par ex. banquets d'associations, combats de coqs, concours de pêche, etc.) (p. 24).

4 Dans l'introduction, l'auteur indique globalement les objectifs de sa recherche en mentionnant tout d'abord les difficultés à appréhender une communauté chinoise qui est loin d'apparaître comme une entité simplement définie par une apparence précise, « homogène, intangible ou nettement distincte » (p. 12). Ensuite, tout en rappelant les éléments historiques concernant la venue des Occidentaux dans la région Pacifique, l'auteur limite la période couverte par sa recherche, du début du XIX siècle à nos jours, et indique les raisons de la venue en Polynésie française des premiers Chinois, principalement du groupe ethnolinguistique hakka. Elle précise également que le terme " Chinois », s'il est « englobant » est également très variable, du fait notamment qu'il y a peu de résidents permanents de nationalité chinoise (p. 20).

5 L'introduction se poursuit avec des définitions, de diaspora, relativement au transnationalisme, et s'opposant à la notion de « fluidité des diasporas et rigidité des nations ", l'auteur inscrit son ouvrage dans une perspective visant à analyser les rapports dynamiques (p. 27) ; de cosmopolitisme, montrant ainsi qu'en Polynésie française, le métissage est généralisé, comme la variabilité des identifications des " Chinois » qui souvent se définissent comme des Polynésiens ; de culture, d'identité, conditionnées par les rapports entre la métropole et la colonie (puis Territoire et Pays d'outre mer) et les rapports sociaux au sein de la société polynésienne, entraînant une succession de développements et de fissions de structures hiérarchiques et de lignages, par le culte des ancêtres, le kasan (p. 34). L'introduction se termine par l'histoire récente et actuelle de la Polynésie française, marquée par une économie de rente (Centre d'expérimentation du Pacifique, CEP, perles, tourisme)

6 La partie 1 s'intitule «La constitution d'une communauté chinoise dans une société multiethnique ». Le premier chapitre porte sur l'histoire de l'immigration chinoise et la création des institutions chinoises. Les facteurs de l'immigration chinoise dépendent de la situation économique et politique de la Chine dès le milieu du XIX siècle (push) et de l'ouverture de la Chine imposée par les Occidentaux pour importer de la main d'œuvre dans leurs colonies (pull). Avec la création d'Atimaono, dans les années 1860, première «importation» de travailleurs chinois dans une plantation de coton qui fait faillite en 1873, quelques centaines seulement restent sur les 1000 Chinois introduits, se réduisant à environ 400 avec les départs et les décès. Il faut attendre le début du $\mathrm{Xx}^{\mathrm{e}}$ siècle pour observer une réelle migration chinoise, (de 1904, début des recensements, à 1947, sur 5404 Chinois, 2927 repartent, 2477 restent, dont des femmes). De 1941 à 1961, environ 3000 Chinois se font immatriculer. L'auteur mentionne trois vagues successives d'immigration : celle des " coolies " d'Atimaono, celle des «aventuriersmarchands» (p. 59) venus avec des objectifs commerciaux et celle des personnes recrutées par ces derniers. Loin d'être homogène, la communauté chinoise s'est stratifiée et segmentée, à la fois selon l'évolution de la situation politique en Chine et celle de la gouvernance en Polynésie, se regroupant en diverses associations, selon trois niveaux : celui englobant toutes les institutions chinoises, puis celui des regroupements sociopolitiques, enfin celui des sociétés secrètes et des associations villageoises cantonaises (p. 66). L'objectif principal des membres de la communauté chinoise est 
avant tout économique et se fonde sur une structure familiale hiérarchisée, une solidarité et une réciprocité entre tongxing (porteurs du même nom) et une relation privilégiée entre familles alliées au travers des différentes associations (p. 86).

7 Le deuxième chapitre concerne l'insertion des Chinois dans les rapports économiques et sociaux avec la société coloniale, et la constitution et différenciation de groupes ethniques dans des niches économiques. Les premiers colons qui se lient à l'aristocratie locale, forment l'élite des « demis », organisent le commerce d'exportation (coton, puis canne à sucre, phosphate, vanille, nacre et coprah) et n'apprécient guère l'arrivée des Chinois qui, rapidement, représentent une sérieuse concurrence à leur oligopole. En effet, dans une économie peu monétarisée, les Chinois s'insèrent par le biais du crédit et se font payer en nature (vanille, nacre). Toutefois, avec un accès au foncier réservé aux seuls colons, l'administration coloniale maintient les frontières ethniques (p. 95). Entre Polynésiens et Chinois, le crédit fonde une relation sur une logique de prodigalité des Polynésiens : dépenses ostentatoires et, pour les Chinois, irrationalité, «argent rapide » (p. 101) et sur une logique de rétention des Chinois : accumulation, rationalité, investissements rémunérateurs. Ces logiques s'étendent à ce que chacun des groupes pense de l'autre et à une appréciation de l'espace différente : les Chinois achètent des parcelles le long de la route et quand il le peuvent, celles au-dessus, où ils installent des magasins et leur famille. Cette société "plurielle» (p. 111) se fige sous l'action de l'administration et sa politique différentielle en matière de nationalité et citoyenneté.

Dans le troisième chapitre, l'auteur montre qu'à la fois extérieur et central, le groupe chinois fait l'objet d'un débat sur l'octroi de la nationalité française, qui devient l'enjeu d'une opposition structurelle entre colons et administration.

Cette vieille opposition, les colons veulent plus de droits politiques, tandis que l'administration impose des taxes, n'empêche pas une élite de se former, qui se retrouve dans les cercles huppés de la capitale, se mélange avec le temps et concentre les charges politiques. Dans ce contexte d'opposition entre colons et administration, la "question chinoise» (p. 120) devient un enjeu politique. Peu à peu, cette rivalité diminue à mesure que l'élite "demie » se constitue, mais les Chinois sont toujours considérés comme des rivaux dans l'entreprise coloniale, et le spectre d'un métissage sino-tahitien est brandi. Au cours de la période 1890-1940, l'administration divise la population en groupes distincts selon des critères raciaux : blancs ou européens, asiatiques et indigènes. Et avec son paternalisme qui lui fait protéger les indigènes, elle trouve chez les Chinois une possibilité d'arbitrage entre les deux autres groupes, tirant le meilleur parti de la présence chinoise, qui devient dès 1919 un "élément indispensable à la prospérité locale » (p. 127), mais qui limite l'immigration dès 1930 et finit par imposer aux Chinois des restrictions de plus en plus sévères en matière d'acquisition de terres, d'activité commerciale, de statut juridique, générant un métissage accru. Pourtant, dès 1946, cette nouvelle génération en appelle à une autonomie accrue, se fondant sur son identité polynésienne, et au sein de laquelle l'élite chinoise revendique la citoyenneté française, et avec elle, tous ceux qui ne sont pas repartis, rebutés par le régime communiste. Avec l'avènement de la Polynésie française en 1956 et la disparition des catégories racialistes en 1973 (p. 144), sur fond de revendication autonomiste, les clivages entre groupes ne reposent plus que sur des dimensions sociale et ethnique. 
10 La partie 2, «Parenté et métissage en Polynésie française » commence par le quatrième chapitre, dans lequel l'auteur analyse l'organisation et le déploiement des familles chinoises à l'Outre-mer. La famille chinoise est un système très flexible, adaptable et solidaire, avec d'un côté, un idéal de la famille étendue, du travail en famille et une concentration du capital (" forces unifiantes ») et, d'un autre, la possibilité de partition de la propriété («fragmentation») entre les fils (p. 150). S'ajustant à la situation transnationale, la famille, la structure de la parenté, est adaptée en fonction de trois variables : politique (invasion japonaise, régime communiste empêchant les pères d'envoyer leur descendance étudier en Chine, ou hiérarchie de la famille dispersant certains membres, frère ainé ou cadet suivant le cas); économique (le boom économique des années 1960 - avec le Centre d'expérimentation du Pacifique, CEP - a contribué à la fragmentation des entreprises familiales); juridique (adaptation de la structure interne d'autorité face aux contraintes par le mariage avec des femmes polynésiennes - françaises - ou la non-reconnaissance des filles sinon par la mère ou des amis polynésiens, ce qui ne va pas toujours sans tensions). Avec un groupe de descendance chevauchant la Chine et Tahiti, une adaptation aux nouvelles opportunités économiques des années 1960 (СEP) et des biens commerciaux et immobiliers au nom des femmes et des filles, la famille chinoise se fragmente mais, solidaire, elle repose toutefois, pour certaines d'entre elles, sur des lignages organisés autour du culte des ancêtres. Le kasan (p. 162) contribue à la formation de lignages dont deux types existent à Tahiti : l'un, fondé sur l'héritage, regroupant la famille privée, le kasan domestique (p. 176), et l'autre, fondé sur l'ensemble des porteurs du même nom, le kasan clanique (180).

11 Dans le cinquième chapitre, illustré par des récits de vie et des schémas de parenté, l'auteur étudie la place des femmes dans les familles chinoises. Selon leurs modalités d'échange, les deux groupes, chinois et polynésien, tous deux exogames (p. 188), cherchent des unions matrimoniales en dehors de leur famille ou leur clan, d'autant que, au début de l'immigration, ce sont surtout des hommes qui arrivent en Polynésie. Et malgré la préférence des Chinois pour des mariages avec des Chinoises, les Polynésiennes entrent dans les familles chinoises selon des échanges complexes fondés sur une structure patrilatérale. Les femmes polynésiennes, si elles ne sont pas «incorporées » à la famille chinoise, y ont une place permanente du fait des liens de réciprocité, distinguée selon deux logiques complémentaires: d'une part, comme génitrices d'un nouveau membre masculin de la lignée (et comme force de travail) et, d'autre part, selon leur statut de mère et leur autonomisation (sortie de l'économie familiale) (p. 221). Ainsi, à une idéologie agnatique (de père en fils), se superpose une logique utérine (lien entre la mère et l'enfant) favorisant la fission du lignage et remettant en cause les principes de patrilinéarité et de patrilocalité.

12 Dans le sixième chapitre, l'auteur examine le métissage, ou le conflit des affiliations. Les frontières entre groupes ethniques s'expliquent aussi selon la circulation des enfants issus d'unions «illégitimes» (p. 223) entre Chinois et Polynésiennes engendrant un métissage fondé sur une dialectique d'inclusion et d'exclusion, laquelle ne favorise pas un sentiment total d'appartenance au groupe chinois. Dans le cadre du maintien de lignées distinctes, d'une manière générale, les filles restent dans la famille maternelle, tandis que les garçons réintègrent la famille paternelle (p. 235), dans un contexte où, en Océanie, l'adoption représente une modalité courante de la circulation des enfants, tandis que pour les Chinois, elle repose sur deux impératifs contradictoires 
: perpétuation du culte ancestral et préservation des lignées agnatiques. L'exclusion des filles et la réintégration des fils, de même que la formulation de l'appartenance à tel ou tel groupe, sont illustrées et analysées avec des cas concrets, permettant de mieux cerner leur complexité et de définir un métissage fondé sur une situation plutôt conflictuelle qu'harmonieuse. Le métissage, issu d'une coexistence entre lignées utérines et agnatiques distinctes, engendre des logiques concurrentes et des entités sociales mêlées. Et parfois, comme le montrent les exemples de récits de vie cités, groupes issus des liens du sang et groupes issus d'un même ancêtre peuvent faire l'objet de situations paradoxales, comme celle d'un père chinois qui ne reconnaît pas ses enfants, mais qui inscrit ses biens au nom de la mère afin que les enfants puissent en hériter (p.255), ou encore celle de lignées chinoise et polynésienne qui se rassemblent autour du kasan (p. 258), sans toutefois s'accorder et donnant lieu à des conflits jusque dans la sphère publique.

13 Vient la partie 3: Multiethnicité et autochtonie. Dans le septième chapitre, l'auteur analyse la restructuration contemporaine de la communauté chinoise. En interrogeant des membres de la communauté chinoise, l'auteur évoque les divisions au sein de la communauté et les conflits de mémoire ayant entraîné une division entre les individus se réclamant d'une identification à la condition de Chinois d'Outre-mer et ceux affichant une ethnicité symbolique chinoise. Le temple de Kanti est révélateur de ces différences, dans la manière d'interpréter le passé et de construire une mémoire communautaire, entre "passé coolie» et "cosmopolitisme» (pp. 280-281). Ou encore, l'exemple du terrain consulaire qui divise la communauté depuis plus de trente ans, non seulement entre les tenants de chacune des «deux Chines» (Taïwan et République populaire) (p. 283), mais aussi concernant le droit de propriété du terrain sur lequel se trouve le consulat. L'auteur s'interroge ensuite sur la transformation du rapport à la Chine et l'ethnicité symbolique et montre que parmi les fractures du lien avec la Chine, la question des ancêtres joue un rôle important, les individus des générations actuelles les situant en Polynésie, de même que la question de la langue hakka qui n'est plus parlée par les générations les plus jeunes, ou celle de la nationalité française, aujourd'hui acquise, normalisant le statut juridique, ou encore celle de la nouvelle relation économique avec la Chine (sud-est, là-même d'où étaient parti les premiers émigrants, et où ont été constituées les premières zones économiques spéciales, ZES), comme la dissolution des villages d'origine dans les villes nouvelles (p. 298). De même, le champ associatif est révélateur des changements du rapport avec la Chine, l'ancienne appartenance prescrite devenant volontaire, mettant en avant une affirmation individuelle quant à l'identité chinoise et une implication dans les activités de la communauté, entraînant un « renouveau culturel chinois » (p. 308).

Dans le huitième chapitre, l'auteur analyse l'ethnicisation du politique et la différenciation des identifications. Dès la fin des années 1960, quelques Chinois se lancent en politique (après avoir été naturalisés français), puis avec l'émergence d'associations culturelles chinoises, se créent des partis politiques aux objectifs variés. Aujourd'hui, il ne subsiste qu'un parti chinois occupant la scène publique (p. 315), lequel marque la différenciation ethnique comme les représentations collectives, notamment avec l'aide des associations culturelles chinoises et donne lieu à une instrumentalisation jusque dans la sphère diplomatique. En 2002, le voyage de Gaston Flosse en Chine, avec pour objectif de négocier des affaires économiques, n'a pas rencontré le succès économique escompté, mais il n'en démontre pas moins que l'ethnicité est instrumentalisée afin de "renforcer les liens historiques entre la Polynésie française et la Chine» (p. 321), dans un contexte focalisé depuis les années 
1980 sur une alternative autonomie/indépendance, où la présence chinoise est associée à une domination économique. Les processus d'identification ont évolué différemment selon le type de socialisation et de milieu socio-économique auxquels les individus ont été confrontés: sentiment d'appartenance locale pour ceux qui ont évolué dans un milieu polynésien, notamment en dehors de Tahiti, à l'échelle du Territoire, et au-delà pour ceux qui ont fait des études et qui ont une identité « cosmopolite» (p. 335).

Dans le neuvième et dernier chapitre, l'auteur se penche sur les dilemmes individuels et l'ambiguïté des identifications. Ce dernier chapitre analyse le métissage à partir de récits de vie d'individus élevés par leur père chinois et leur mère polynésienne, mettant en lumière leur construction culturelle, dans un cadre de ruptures et de discontinuités des trajectoires liées aux structures sociales. Dilemmes identitaires et socialisations concurrentes reposent sur un antagonisme entre deux lois intervenant dans la socialisation, celle du père chinois, fondée sur la valorisation de la culture chinoise, le travail et la discipline, et celle de la mère polynésienne fondée sur l'éducation dans un milieu concurrent à celui de l'école chinoise, donc la langue tahitienne, et sur l'église (p. 361). Toutefois, reposant sur le renversement de stigmate, ces dilemmes identitaires peuvent être surmontés par la construction d'une nouvelle identification mā'ohi dépassant le conflit entre identité chinoise et identité polynésienne (p. 363). L'individu, relevant d'une tendance ou de l'autre, prend ses distances en affirmant une identité polynésienne et indépendantiste.

Dans sa conclusion, l'auteur rappelle que les Chinois de Polynésie française ont peu à peu perdu leur caractéristique diasporique, notamment depuis leur autonomisation, et qu'avec le métissage, à la troisième ou quatrième génération, apparaissent des identités soit locale, soit cosmopolite, puis entre elles une nouvelle identité mā'ohi, issue d'une identification choisie plutôt qu'assignée dans un contexte de concurrence des idéologies chinoise et polynésienne. Généralisant et s'opposant à une définition univoque de l'individu, l'auteur propose la notion de « sujet clivé » (p. 380) entre deux modes d'identification, refusant une explication reposant sur le seul facteur de l'origine et des valeurs chinoises.

Enfin, cet ouvrage montre l'importance d'une démarche empirique, qualitative, pour analyser une situation locale, comme celle de la présence chinoise en Polynésie française, en saisir toute la complexité et, grâce à un travail anthropologique, dégager une généralisation, celle du migrant chinois, l'entrepreneur par excellence, audacieux économiquement et adaptable socialement. 